BRAVZULIAN JOURNAL

OF MEDICAL AND BIOLOGICAL RESH.ARCH

www.bjournal.com.br
ISSN 0100-879X

Volume 44 (6) 497-605 June 2011

BIOMEDICAL SCIENCES

AND

CLINICAL INVESTIGATION

Braz J Med Biol Res, June 2011, Volume 44(6) 592-597

doi: 10.1590/S0100-879X2011007500059

Do whole-body vibration exercise and resistance exercise modify concentrations of salivary cortisol and immunoglobulin A?

H. Roschel, R. Barroso, M. Batista, C. Ugrinowitsch, V. Tricoli, F. Arsati, Y.B. Lima-Arsati, V.C. Araújo and A. Moreira

The Brazilian Journal of Medical and Biological Research is partially financed by

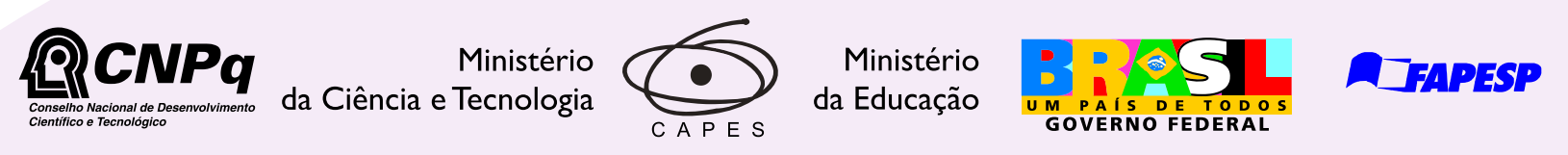

Institutional Sponsors
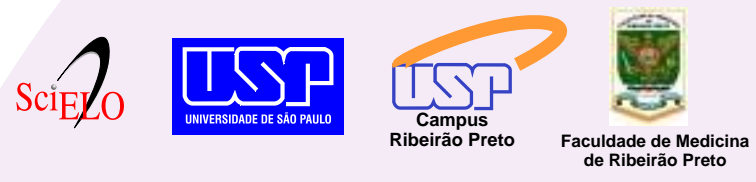

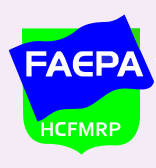

Ф SHIMADZU

GE Healthcare
Hotsite of proteomics metabolomics developped by:

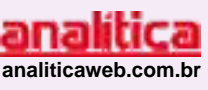

$\underset{\text { SCIENTIFIC }}{\text { Thermo }}$ 


\title{
Do whole-body vibration exercise and resistance exercise modify concentrations of salivary cortisol and immunoglobulin A?
}

\author{
H. Roschel ${ }^{1}$, R. Barroso ${ }^{1}$, M. Batista ${ }^{1}$, C. Ugrinowitsch ${ }^{1}$, V. Tricoli ${ }^{1}$, \\ F. Arsati ${ }^{2}$, Y.B. Lima-Arsati ${ }^{2}$, V.C. Araújo ${ }^{3}$ and A. Moreira ${ }^{1}$ \\ ${ }^{1}$ Escola de Educação Física e Esporte, \\ Universidade de São Paulo, São Paulo, SP, Brasil \\ 2Departamento de Ciências Fisiológicas, ${ }^{3}$ Departamento de Patologia Oral, São Leopoldo Mandic, \\ Centro de Pesquisa Odontológica, Campinas, SP, Brasil
}

\begin{abstract}
A single bout of resistance exercise (RE) induces hormonal and immune responses, playing an important role in a long-term adaptive process. Whole-body vibration (WBV) has also been shown to affect hormonal responses. Evidence suggests that combining WBV with RE may amplify hormonal and immune responses due to the increased neuromuscular load. Therefore, the aim of this study was to evaluate salivary cortisol (Scortisol) and salivary $\operatorname{lgA}(\mathrm{S} \lg \mathrm{A})$ concentrations following a RE session combined or not with WBV. Nine university students $(22.9 \pm 5.1$ years, $175.8 \pm 5.2 \mathrm{~cm}$, and $69.2 \pm 7.3 \mathrm{~kg})$ performed five sets of squat exercise $(70 \%$ one-repetition-maximum) combined $(\mathrm{R}+\mathrm{V} 30)$ or not $(\mathrm{R})$ with WBV at $30 \mathrm{~Hz}$. Saliva samples were obtained before and after exercise. Subjects also rated their effort according to the Borg CR-10 scale (RPE). Data were analyzed by a mixed model. RPE was higher after R+V30 (8.3 \pm 0.7$)$ compared to R (6.2 \pm 0.7$)$. However, Scortisol (pre: $10.6 \pm 7.6$ and 11.7 \pm 7.6 , post: $8.3 \pm 6.3$ and $10.2 \pm 7.2 \mathrm{ng} / \mathrm{mL}$ for $R$ and $R+V 30$, respectively) and SIgA concentrations (pre: $98.3 \pm 22.6$ and 116.1 \pm 51.2 , post: $116.6 \pm 64.7$ and $143.6 \pm 80.5 \mu \mathrm{g} / \mathrm{mL}$ for $R$ and $R+V 30$, respectively) were unaffected. No significant correlations were observed between Scortisol and RPE $(r=0.45, P=0.22 ; r=0.30, P=0.42$, for $R$ and $R+V 30$, respectively). On the basis of these data, neither protocol modified salivary cortisol or IgA, although RPE was higher after R+V30 than R.
\end{abstract}

Key words: Upper respiratory tract infection; Hormonal responses; Immune responses; Neuromuscular training

\section{Introduction}

An association between exercise and the incidence of upper respiratory tract infections (URTI) has been proposed by Nieman (1). Indeed, a decrease in the secretion rate of salivary markers of mucosal immunity, particularly salivary immunoglobulin $\mathrm{A}(\mathrm{S} \lg \mathrm{A})$, has been related to an increased risk of URTI episodes (2). However, information about the SIgA secretion response after exercise is contradictory. For instance, an acute decrease in SIgA concentration occurs after intense endurance exercise (3), whereas intermittent sport-based exercises have been shown to promote either no changes $(4,5)$ or an increase in SIgA levels $(6)$.

Thus, while it seems that exercise has an impact on the mucosal immunity response, the effect of short-duration moderate-intensity resistance exercise (RE) bouts on SIgA secretion has not been reported $(7,8)$. The literature has shown that a single bout of RE is able to elicit an acute increase in circulating cortisol concentrations $(2,9-11)$ and that salivary cortisol (Scortisol) concentration is related to the suppression of SIgA secretion (12).

Whole body vibration (WBV) has been recently suggested as an alternative way to perform RE. During a WBV session, subjects exercise on a vibrating platform that produces sinusoidal oscillations. Interestingly, it has been advocated that performing RE on a vibrating platform induces greater neuromuscular and hormonal responses than RE alone by providing a more intense stimulus $(13,14)$. Accordingly, Kvorning et al. (13) demonstrated that combining WBV with RE promotes greater changes in cortisol secretion when compared to a similar protocol of $\mathrm{RE}$ alone. Therefore, it seems reasonable to suggest that

Correspondence: H. Roschel, Av. Prof. Mello Moraes, 65, 05508-030 São Paulo, SP, Brazil. Fax: +55-11-3813-5091.

E-mail: hars@usp.br

Received December 2, 2010. Accepted April 28, 2011. Available online May 13, 2011. Published June 13, 2011. 
the combination of RE with WBV might produce a greater suppression of SIgA secretion than RE alone.

To date, no study has been conducted to evaluate the acute Scortisol and SIgA responses to a single bout of a hypertrophy-oriented RE combined with WBV. Therefore, the aim of the present study was to measure Scortisol and SIgA concentrations following a session of RE alone and an RE session combined with WBV in healthy young adult males. We expected that the addition of WBV to the RE protocol would result in a greater training stimulus than provided by RE alone, thus inducing a greater increase in Scortisol concentration and a greater decrease in SIgA concentration.

\section{Material and Methods}

A cross-over design was used to examine if combining RE with WBV would induce greater hormonal and acute immune responses than $\mathrm{RE}$ alone. For this purpose, participants were required to perform two bouts of a hypertrophyoriented RE in a balanced and randomized order. Each experimental session was performed on a different day, with session separated by a 7-day interval. The RE bout consisted of a loaded back-squat exercise (R). The other experimental session comprised the addition of WBV at $30 \mathrm{~Hz}$ to the $\mathrm{R}$ protocol $(\mathrm{R}+\mathrm{V} 30)$. Samples of saliva were obtained before and immediately after all experimental sessions and analyzed for Scortisol and SIgA concentrations. In addition, to determine the intensity of each experimental protocol, subjects were asked to report a rating of perceived exertion (RPE) at the end of each session to determine the exercise intensity. All experimental sessions took place during the same time of day (in the afternoon, between 2:00 and 5:00 pm) and had the same duration (approximately $18 \mathrm{~min})$.

\section{Subjects}

Nine healthy male university students $(22.9 \pm 5.1$ years, $175.8 \pm 5.2 \mathrm{~cm}$, and $69.2 \pm 7.3 \mathrm{~kg}$ ) volunteered to participate in the study. The participants had been involved in resistance training for at least 6 months ( $10 \pm 3$ months) and were free from musculoskeletal disorders. None of the participants had used any dietary supplements or medications for at least 12 months prior to this investigation. Participants were informed of the experimental risks and signed an informed consent form prior to the investigation. The study was approved by the Ethics Committee of the Escola de Educação Física e Esporte, Universidade de São Paulo.

\section{Familiarization and testing session}

All participants completed two sessions of familiarization with the maximum dynamic strength test (i.e., back-squat 1-RM test) protocol, and two additional sessions of familiarization with the WBV protocol (i.e., three sets of light-loaded back-squat exercise on the vibrating platform).
Two days after the familiarization sessions, the maximum dynamic strength of the lower limbs (1-RM) was measured on a Smith machine (Cybex International Inc., USA). The test protocol followed the suggestions proposed by Brown and Weir (15). In short, the participants performed a general warm-up followed by lower limb light-stretching exercises and two squat exercise warm-up sets. For the first set, the participants were asked to perform 5 repetitions with $50 \%$ of their estimated 1-RM. The second set comprised the completion of three repetitions with $70 \%$ of their estimated 1-RM. A 3-min interval was allowed between sets. After the second warm-up set, the subjects rested for $3 \mathrm{~min}$. Then, they were allowed up to five trials to achieve the 1-RM load (i.e., maximum weight that could be lifted once with the proper technique), with a 3-min interval between trials. The maximal load lifted during the 1-RM test was used to determine the external load for all experimental conditions.

\section{Saliva collection}

Saliva samples were collected before and immediately after the exercise protocols. The participants were instructed to avoid any food intake, drinking hot fluids, brushing their teeth $2 \mathrm{~h}$ before the assessment, and to refrain from exercising for $48 \mathrm{~h}$ prior to the experimental sessions. Participants were instructed to maintain their normal dietary intake on each testing day. The subjects were asked to remain seated in the laboratory area for $\mathbf{4 5}$ min prior to provide the resting sample. All participants remained in a seated position, with their eyes open and head tilted slightly forward while making minimal orofacial movement. Unstimulated saliva was collected into sterile $15-\mathrm{mL}$ centrifuge tubes over a 5-min period. Immediately after collection, the saliva samples were frozen and stored at $-80^{\circ} \mathrm{C}$ until assays for cortisol and SIgA concentrations were performed.

\section{Measurement of Scortisol and SIgA concentration}

The concentration of Scortisol in saliva was measured by enzyme-linked immunosorbent assay (ELISA, Cortisol - Direct Salivary EIA; ALPCO Diagnostics, USA) as described in a previous report by our group (16). Briefly, saliva samples were thawed and centrifuged at $1630 \mathrm{~g}$ for $20 \mathrm{~min}$ and then, cortisol concentration was measured by ELISA (ALPCO Diagnostics). From a calibration curve (absorbance vs cortisol concentration of the calibrators), the concentration of cortisol $(\mathrm{ng} / \mathrm{mL})$ in each sample was interpolated. All assays were carried out on the same day.

SIgA concentration was measured by a similar procedure (ELISA Starter Accessory Package and Human IgA ELISA Quantitation Set; \#E101 and \#E80-102, respectively; Bethyl Laboratories, USA). Briefly, a 96-well microtiter plate was coated with $100 \mu \mathrm{L} 10 \mu \mathrm{g} / \mathrm{mL}$ goat antihuman IgA diluted in coating buffer $(0.05 \mathrm{M}$ carbonate-bicarbonate, $\mathrm{pH} 9.6)$ and incubated at room temperature $\left(20-25^{\circ} \mathrm{C}\right)$ for $1 \mathrm{~h}$. After incubation, the plate was aspirated and washed 
five times with $250 \mu \mathrm{L}$ wash solution (50 mM Tris-buffered saline, $\mathrm{pH} 8.0,0.05 \%$ Tween 20 ) to remove all unbound substances. Then, $200 \mu \mathrm{L}$ ELISA blocking solution ( $50 \mathrm{mM}$ Tris, $0.14 \mathrm{M} \mathrm{NaCl}, 1 \% \mathrm{BSA}, \mathrm{pH} 8.0$ ) was added to each well and incubated at room temperature for $30 \mathrm{~min}$. After incubation, the plate was aspirated and washed five times with $250 \mu \mathrm{L}$ wash solution. Saliva samples were thawed and centrifuged at $1630 \mathrm{~g}$ for $10 \mathrm{~min}$, and the supernatant was diluted 1:2000 in ELISA sample/conjugate diluent (50 $\mathrm{mM}$ Tris, $0.14 \mathrm{M} \mathrm{NaCl}, 1 \% \mathrm{BSA}, 0.05 \%$ Tween 20 ). Then, $100 \mu \mathrm{L}$ of the calibrators and diluted saliva samples were added to the wells and the plates were incubated for $1 \mathrm{~h}$ at room temperature $\left(25^{\circ} \mathrm{C}\right)$. After incubation, the plate was aspirated and washed five times with $250 \mu \mathrm{L}$ wash solution to remove all unbound substances. Then, $100 \mu \mathrm{L}$ peroxidase-labeled mouse anti-lgA conjugate was added to each well. After incubation for $1 \mathrm{~h}$ at room temperature, the plate was aspirated and washed five times with 250 $\mu \mathrm{L}$ wash solution to remove all unbound substances. After washing, $100 \mu \mathrm{L}$ tetramethylbenzidine substrate solution was added and the plates were incubated for $15 \mathrm{~min}$ in the dark at room temperature. Finally, $100 \mu \mathrm{L}$ of the stop solution (2 $\mathrm{M} \mathrm{H}_{2} \mathrm{SO}_{4}$ ) was added to the wells and absorbance was read on the plate reader at $450 \mathrm{~nm}$. From a calibration curve (absorbance vs IgA concentration of the calibrators) the concentration of SIgA $(\mathrm{ng} / \mathrm{mL})$ in each sample was interpolated. The results are reported as $\mu \mathrm{g} / \mathrm{mL}$. The SIgA secretion rate $(\mu \mathrm{g} / \mathrm{min})$ was calculated by multiplying the absolute SIgA concentration by the salivary flow rate $(\mathrm{mL} /$ min). The salivary flow rate was determined by dividing the measured volume of saliva collected by the time of the sampling period.

In our laboratory, the test-retest correlation coefficients for SIgA and Scortisol are 0.96 and 0.98 , respectively. The coefficient of variation of the intra-assay measurements (duplicates) was $7.0 \%$ for SIgA (SD: $7.0 \mu \mathrm{g} / \mathrm{mL}$ ) and $9.0 \%$ for Scortisol (SD: $0.68 \mathrm{ng} / \mathrm{mL}$ ). The saliva samples of each participant were analyzed in the same assay and on the same day (using the same kit) in order to eliminate interassay variance. The sensitivity of the cortisol assay was $1 \mathrm{ng} / \mathrm{mL}$ and the detection limit for SlgA was $1.03 \mathrm{ng} / \mathrm{mL}$. Cortisol and SIgA ranges were 1.0-100 ng/mL and 7.8-500 $\mathrm{ng} / \mathrm{mL}$, respectively. The $\mathrm{R}^{2}$ of the standard curve was 0.99 for both assays.

\section{Exercise protocols}

RE protocol. Aconventional loaded back-squat exercise was performed on a Smith-Machine equipment (Cybex International Inc., USA). A consistent body position and foot placement across experimental sessions were guaranteed with measuring tape fixed to the bar and to the ground, respectively. In addition, a wooden seat of adjustable height was placed behind the subjects to keep the bar displacement and the knee angular displacement constant. The exercise protocol consisted of five sets of 10 repetitions with $70 \%$
1-RM. The subjects rested for 2 min between sets.

RE combined with WBV protocols. The combination of RE and WBV consisted of performing the squat exercise as mentioned above while standing on the vibrating platform (Fitvibe 600 , Gmna Uniphy, Belgium). The WBV platform frequency was set at $30 \mathrm{~Hz}(\mathrm{R}+\mathrm{V} 30)$ and amplitude at 2-4 mm.

$R P E$. RPE was recorded for all subjects using the Borg CR-10 scale to determine the exercise bout intensity. Assessments were made immediately after the exercise and before the saliva collection. Participants were asked to rate the difficulty of performing the exercise using a 10-point scale.

\section{Statistical analysis}

Data normality was determined by the Shapiro-Wilk test and by standard visual inspection. All variables presented a normal distribution. A mixed model was used for each dependent variable, with protocol and time considered to be fixed factors, and subjects to be random factors. Whenever a significant F-value was obtained, a post hoc test with Tukey adjustment was performed for multiple comparison purposes. The Pearson correlation coefficient was used to test the relationship between pre- and postexercise values of SIgA and Scortisol with RPE. The level of significance was set at $P \leq 0.05$. All data are reported as means \pm SEM.

\section{Results}

Scortisol concentration did not differ between the experimental conditions at rest before exercise $(10.6 \pm 7.6$ and $11.7 \pm 7.6 \mathrm{ng} / \mathrm{mL}$ for $\mathrm{R}$ and $\mathrm{R}+\mathrm{V} 30$, respectively; $\mathrm{P}=$ 0.98). Also, the Scortisol response to exercise was not affected by the exercise protocols $(8.3 \pm 6.3, P=0.78$ and $10.2 \pm 7.2 \mathrm{ng} / \mathrm{mL}, \mathrm{P}=0.92$, for $\mathrm{R}$ and $\mathrm{R}+\mathrm{V} 30$, respectively; Figure 1).

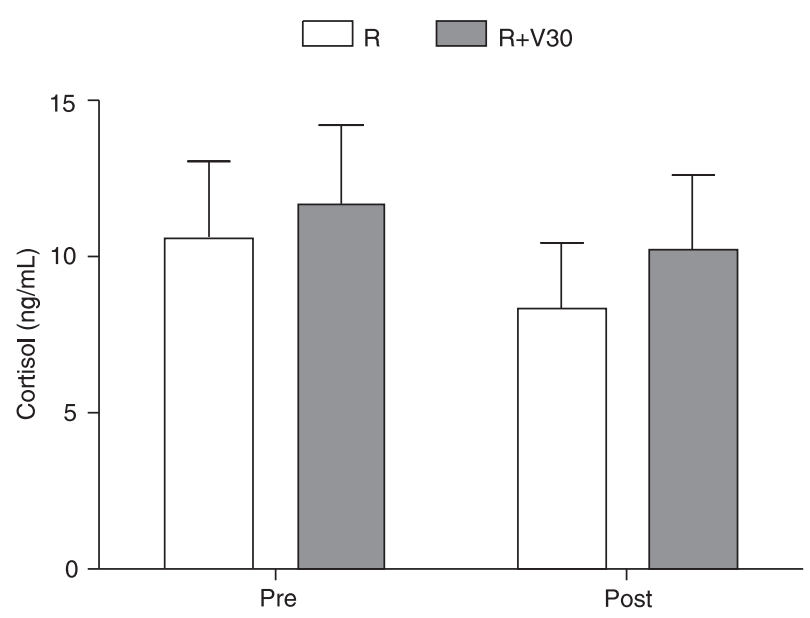

Figure 1. Salivary cortisol $(\mathrm{ng} / \mathrm{mL})$ before and immediately after the two exercise conditions. 
SIgA concentration $(98.3 \pm 22.6$ and $116.1 \pm 51.2 \mu \mathrm{g} /$ $\mathrm{mL}$ for $\mathrm{R}$ and $\mathrm{R}+\mathrm{V} 30$, respectively; $\mathrm{P}=0.92)$ and secretion rate $(28.2 \pm 27.4$ and $27.1 \pm 22.7 \mu \mathrm{g} / \mathrm{min}$ for $\mathrm{R}$ and $\mathrm{R}+\mathrm{V} 30$, respectively; $P=0.99)$, and the salivary flow rate $(0.32 \pm$ 0.32 and $0.37 \pm 0.42 \mathrm{~mL} / \mathrm{min}$ for $\mathrm{R}$ and $\mathrm{R}+\mathrm{V} 30$, respectively; $\mathrm{P}=0.97$ ) did not differ between the two conditions at rest before exercise. Furthermore, no pre- to post-exercise differences were found for SIgAconcentration (116.6 $\pm 64.7, P$ $=0.84$ and $143.6 \pm 80.5 \mu \mathrm{g} / \mathrm{mL}, P=0.62$, for $R$ and $R+V 30$, respectively), SIgA secretion rate $(29.6 \pm 31.0, P=0.99$ and $25.2 \pm 23.2 \mu \mathrm{g} / \mathrm{min}, P=0.98$, for $R$ and $R+V 30$, respectively) or salivary flow rate $(0.24 \pm 0.20, P=0.80$ and $0.25 \pm 0.33$ $\mathrm{mL} / \mathrm{min}, \mathrm{P}=0.50$, for $\mathrm{R}$ and $\mathrm{R}+\mathrm{V} 30$, respectively) in response to either exercise condition tested (Figure 2).

Mean RPE scores were $6.2 \pm 0.7$ and $8.3 \pm 0.7$ for the $\mathrm{R}$ and $\mathrm{R}+\mathrm{V} 30$ protocols, respectively. Significant differences were observed between the $R$ and the $R+V 30(P=0.04)$ protocols. Moreover, no significant correlations were found between RPE and the changes in the salivary parameters $(P>0.05)$.

\section{Discussion}

The aim of the present study was to determine the Scortisol and SIgA responses to a single bout of RE alone or combined with WBV at $30 \mathrm{~Hz}$. The biochemical findings of the present investigation were that Scortisol and SIgA were not affected by either exercise protocol. Nonetheless, we found greater RPE after the R+V30 protocol with these data obtained by a questionnaire completed by the subjects.

The cortisol responses observed in the present study were not consistent with previous reports that have shown acute increases in serum cortisol $(17,18)$ or Scortisol $(19,20)$ concentrations after a single bout of a hypertrophy-oriented exercise. Crewther et al. (19) suggested that the increase in cortisol concentration is related to the total volume of RE (i.e., number of sets $x$ external load). These investigators found an increase in Scortisol after a hypertrophy-oriented training routine, whereas maximal strength- and powertraining routines, which comprise a lesser total exercise volume, produced no significant changes in Scortisol. Mulligan et al. (17) corroborated these findings when they observed greater increases in cortisol after multiple sets rather than a single set of hypertrophy-oriented RE. In fact, acute increases in cortisol have been demonstrated after $\mathrm{RE}$ routines involving multiple exercises and/or involving a large muscle mass $(9,17-19,21)$.

Thus, one may speculate that the absence of an increase in Scortisol in the present study after the $\mathrm{R}$ protocol is related to the low volume of exercise performed in comparison with previous studies. Crewther et al. (19) observed increases (ranging from $47-290 \%$ ) in Scortisol after 10 sets of 10 reps with $75 \% 1-\mathrm{RM}$ on the supine squat machine, suggesting that increasing the number of sets (i.e., exercise volume) can affect the cortisol response. However, Smilios et al.
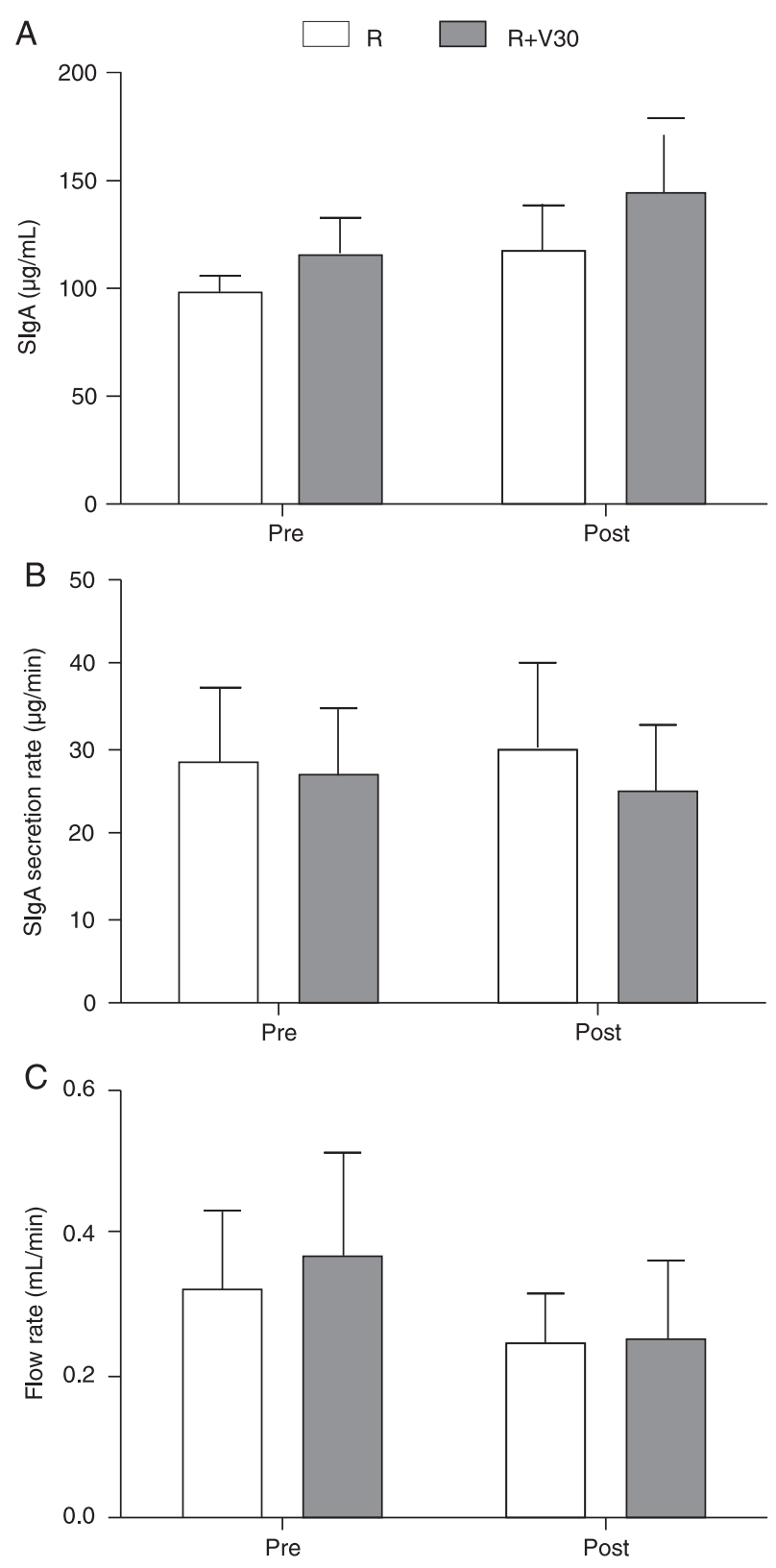

Figure 2. $A$, Salivary immunoglobulin $A(S \lg A)$ concentration $(\mu \mathrm{g} / \mathrm{mL}) ; B$, SlgA secretion rate $(\mu \mathrm{g} / \mathrm{min}) ; C$, salivary flow rate $(\mathrm{mL} / \mathrm{min})$, before and immediately after the two exercise conditions.

(22) did not observe additional changes in serum cortisol concentrations when the number of sets was increased from four to six (approximately $30 \%$ for both groups). Similarly, Hough et al. (23) did not find significant increases in Scortisol after eight sets of $10 \mathrm{RM}$ of the squat exercise. It is important to emphasize that these investigators performed the assessments at 10,20,30,40,50, and 60 min after the exercise bout compared to the immediately after exercise 
assessments performed in the present study.

We also suggest that there may be a delay between the peak rises in serum and saliva cortisol concentrations. As a consequence, we would not be able to detect the changes in Scortisol concentration immediately after the exercise bout. However, McGuigan et al. (24) obtained saliva samples at the beginning, immediately after and 30 min after the RE bouts, and detected a significant increase in Scortisol level only immediately after the high intensity exercise session, with no significant differences between pre- and 30-min post-values. Moreover, Bottaro et al. (25) did not detect any changes in serum cortisol levels in young women performing four lower-body exercises until failure (10 RM) with rest intervals of either 30 or $120 \mathrm{~s}$ between sets. Blood samples for cortisol determinations were drawn before and immediately after the exercise and at 5, 15, and 30 min of recovery.

Another possible explanation for the lack of change in Scortisol is the exercise intensity used in the present study. Participants performed the RE against an external load of $70 \%$ 1-RM, while Kraemer et al. (18) adopted a 10-RM intensity. According to Shimano et al. (26), a 10-RM load in the back-squat exercise corresponds to a relative load of approximately $80 \% 1-\mathrm{RM}$, which suggests that in our study the $\mathrm{R}$ protocol was performed with a submaximal load. However, it is of paramount importance to note that the participants were not able to perform five sets of $10 \mathrm{RM}$ of the squat exercise on the WBV platform. Our RPE values after the $\mathrm{R}+\mathrm{V} 30$ conditions agree with these findings and further support the proposed exercise protocol.

RPE has been considered to be a reliable tool for the assessment of RE intensity $(27,28)$. In the present study, greater RPE was observed after R+V30 compared with R, suggesting that the addition of WBV provided a more intense stimulus than RE alone. However, the increased stimulus provided by the addition of WBV was not sufficient to affect either the Scortisol or the SIgA secretion response.

There are two possible explanations for the greater RPE values observed after the WBV protocols. The first is that RPE is considered to be a psychophysiological phenomenon. For example, Morgan (29) proposed that the effort perception should be understood as a "gestalt" within a construct with numerous physiological and psychological traits, as well as the individual's past experiences. Past experiences could be an important feature in our study. Even though the participants performed two additional sessions of familiarization with the WBV protocol before

\section{References}

1. Nieman DC. Exercise, infection, and immunity. Int $J$ Sports Med 1994; 15 (Suppl 3): S131-S141.

2. Ahtiainen JP, Pakarinen A, Alen M, Kraemer WJ, Hakkinen $\mathrm{K}$. Short $v s$ long rest period between the sets in hypertrophic the beginning of the actual assessments, it is reasonable to suggest that their limited experience with the WBV platform may have produced the greater RPE values, even in the absence of the expected increments in the biological marker of stress (Scortisol). Moreover, some studies have reported inconsistent and largely negative results concerning the psycho-endocrine covariance considering a variety of stressors and subjects $(30,31)$, which may explain, at least in part, the non-significant relationship between RPE and Scortisol changes. The second explanation is related to the activity of the proprioceptors. As RPE seems to be modulated by the afferent volley, it is possible that the WBV may increase the firing rate of cutaneous, muscle, tendon, and joint receptors $(32,33)$, increasing the overall perception of effort during exercise.

The combination of RE with WBV protocols has been suggested as a valid form of increasing exercise intensity $(13,14)$. Similar to the findings reported here, Kvorning et al. (13) did not find increases in cortisol after RE alone. On the other hand, in contrast to our findings, the authors reported a significant increase in serum cortisol levels after combining RE with WBV. The difference between our results and those reported by Kvorning et al. (13) may be possibly explained by the vibration frequency adopted in each study. While the Kvorning et al. (13) tested vibration frequencies between 20 and $25 \mathrm{~Hz}$ (13), we used a slightly higher WBV frequency (i.e., $30 \mathrm{~Hz}$ ).

In addition to the difference in the vibration frequency regimen, Kvorning et al. (13) used a platform that delivered vibration to the body by an alternating rotation around the central axis of the platform. In contrast, we applied WBV through a platform, which produces oscillation in the vertical direction, accelerating both sides of the platform simultaneously. Pel et al. (34) showed that the rotational oscillating platform produces twice the vertical acceleration compared to the vertical oscillation platform when both devices are set at the frequencies of 25 and $30 \mathrm{~Hz}$. It is possible that the vertical acceleration produced in our study was insufficient, which may explain our discrepant findings.

On the basis of these data, neither protocol modified salivary cortisol or IgA, although RPE was higher after $\mathrm{R}+\mathrm{V} 30$ than $\mathrm{R}$ alone.

\section{Acknowledgments}

H. Roschel is supported by FAPESP (\#2010/51428-2). resistance training: influence on muscle strength, size, and hormonal adaptations in trained men. J Strength Cond Res 2005; 19: 572-582.

3. MacKinnon LT, Jenkins DG. Decreased salivary immuno- 
globulins after intense interval exercise before and after training. Med Sci Sports Exerc 1993; 25: 678-683.

4. Sari-Sarraf V, Reilly T, Doran DA. Salivary IgA response to intermittent and continuous exercise. Int J Sports Med 2006; 27: 849-855.

5. Koch AJ, Wherry AD, Petersen MC, Johnson JC, Stuart MK, Sexton WL. Salivary immunoglobulin A response to a collegiate rugby game. J Strength Cond Res 2007; 21: 86-90.

6. Sari-Sarraf V, Reilly T, Doran D, Atkinson G. Effects of repeated bouts of soccer-specific intermittent exercise on salivary IgA. Int J Sports Med 2008; 29: 366-371.

7. Neves Sda C Jr, Lima RM, Simoes HG, Marques MC, Reis VM, de Oliveira RJ. Resistance exercise sessions do not provoke acute immunosuppression in older women. $J$ Strength Cond Res 2009; 23: 259-265.

8. McDowell SL, Weir JP, Eckerson JM, Wagner LL, Housh $T J$, Johnson GO. A preliminary investigation of the effect of weight training on salivary immunoglobulin A. Res Q Exerc Sport 1993; 64: 348-351.

9. Kraemer WJ, Ratamess NA. Hormonal responses and adaptations to resistance exercise and training. Sports Med 2005; 35: 339-361.

10. Linnamo V, Pakarinen A, Komi PV, Kraemer WJ, Hakkinen $\mathrm{K}$. Acute hormonal responses to submaximal and maximal heavy resistance and explosive exercises in men and women. J Strength Cond Res 2005; 19: 566-571.

11. Ahtiainen JP, Pakarinen A, Kraemer WJ, Hakkinen K. Acute hormonal and neuromuscular responses and recovery to forced vs maximum repetitions multiple resistance exercises. Int J Sports Med 2003; 24: 410-418.

12. Hucklebridge F, Clow A, Evans P. The relationship between salivary secretory immunoglobulin A and cortisol: neuroendocrine response to awakening and the diurnal cycle. Int $J$ Psychophysiol 1998; 31: 69-76.

13. Kvorning T, Bagger M, Caserotti P, Madsen K. Effects of vibration and resistance training on neuromuscular and hormonal measures. Eur J Appl Physiol 2006; 96: 615-625.

14. Ronnestad BR. Comparing the performance-enhancing effects of squats on a vibration platform with conventional squats in recreationally resistance-trained men. J Strength Cond Res 2004; 18: 839-845.

15. Brown LE, Weir JP. ASEP Procedures recommendation I: Accurate assessment of muscular strength and power. $J$ Exerc Physiol online 2001; 4: 1-21.

16. Moreira A, Arsati F, de Oliveira Lima Arsati YB, da Silva DA, de Araujo V. Salivary cortisol in top-level professional soccer players. Eur J Appl Physiol 2009; 106: 25-30.

17. Mulligan SE, Fleck SJ, Gordon SE, Koziris LP, TriplettMcBride NT, Kraemer WJ. Influence of resistance exercise volume on serum growth hormone and cortisol concentrations in women. J Strength Cond Res 1996; 10: 256-262.

18. Kraemer WJ, Hakkinen K, Newton RU, Nindl BC, Volek JS, McCormick M, et al. Effects of heavy-resistance training on hormonal response patterns in younger vs older men. J Appl Physiol 1999; 87: 982-992.

19. Crewther B, Cronin J, Keogh J, Cook C. The salivary tes- tosterone and cortisol response to three loading schemes. J Strength Cond Res 2008; 22: 250-255.

20. Beaven CM, Gill ND, Cook CJ. Salivary testosterone and cortisol responses in professional rugby players after four resistance exercise protocols. J Strength Cond Res 2008; 22: 426-432.

21. Uchida MC, Crewther BT, Ugrinowitsch C, Bacurau RF, Moriscot AS, Aoki MS. Hormonal responses to different resistance exercise schemes of similar total volume. $J$ Strength Cond Res 2009; 23: 2003-2008.

22. Smilios I, Pilianidis T, Karamouzis M, Tokmakidis SP. Hormonal responses after various resistance exercise protocols. Med Sci Sports Exerc 2003; 35: 644-654.

23. Hough JP, Papacosta E, Wraith E, Gleeson M. Plasma and salivary steroid hormone responses of men to high-intensity cycling and resistance exercise. J Strength Cond Res 2011; 25: 23-31.

24. McGuigan MR, Egan AD, Foster C. Salivary cortisol responses and perceived exertion during high intensity and low intensity bouts of resistance training. J Sport Sci Med 2004; 3: 8-15.

25. Bottaro M, Martins B, Gentil P, Wagner D. Effects of rest duration between sets of resistance training on acute hormonal responses in trained women. J Sci Med Sport 2009; 12: 73-78.

26. Shimano T, Kraemer WJ, Spiering BA, Volek JS, Hatfield $\mathrm{DL}$, Silvestre R, et al. Relationship between the number of repetitions and selected percentages of one repetition maximum in free weight exercises in trained and untrained men. J Strength Cond Res 2006; 20: 819-823.

27. Sweet TW, Foster C, McGuigan MR, Brice G. Quantitation of resistance training using the session rating of perceived exertion method. J Strength Cond Res 2004; 18: 796-802.

28. Day ML, McGuigan MR, Brice G, Foster C. Monitoring exercise intensity during resistance training using the session RPE scale. J Strength Cond Res 2004; 18: 353-358.

29. Morgan WP. Psychological components of effort sense. Med Sci Sports Exerc 1994; 26: 1071-1077.

30. Cohen S, Hamrick N, Rodriguez MS, Feldman PJ, Rabin BS, Manuck SB. The stability of and intercorrelations among cardiovascular, immune, endocrine, and psychological reactivity. Ann Behav Med 2000; 22: 171-179.

31. Oswald LM, Mathena JR, Wand GS. Comparison of HPA axis hormonal responses to naloxone vs psychologicallyinduced stress. Psychoneuroendocrinology 2004; 29: 371388.

32. Cafarelli E. Peripheral contributions to the perception of effort. Med Sci Sports Exerc 1982; 14: 382-389.

33. Rotto DM, Kaufman MP. Effect of metabolic products of muscular contraction on discharge of group III and IV afferents. J Appl Physiol 1988; 64: 2306-2313.

34. Pel JJ, Bagheri J, van Dam LM, van den Berg-Emons HJ, Horemans HL, Stam HJ, et al. Platform accelerations of three different whole-body vibration devices and the transmission of vertical vibrations to the lower limbs. Med Eng Phys 2009; 31: 937-944. 